\title{
Propofol for status epilepticus in pregnancy
}

\begin{abstract}
We present a 31-year-old pregnant, with prior intractable epilepsy, at 20-weeks of gestation, who was admitted with refractory status epilepticus. Unresponsiveness to first and second line anti-seizure therapy necessitated the use of continuous intravenous infusion of Propofol for 48 hours, which led to clinical and electrographic response without adverse squeal for the mother or the fetus.
\end{abstract}

Keywords: Pregnancy, Status Epilepticus, Propofol
Volume 3 Issue 6 - 2015

\author{
Nadya Al Matrooshi,' Norah Ali,' M Samer \\ Abdalla, ${ }^{2}$ Pramod Anidee Pathikkaran, ${ }^{3}$ \\ Shobhit Sinha ${ }^{4}$ \\ 'Department of Medicine, Mafraq Hospital, United Arab \\ Emirates \\ ${ }^{2}$ Division of Critical Care, Mafraq Hospital, United Arab \\ Emirates \\ ${ }^{3}$ Section of Neurophysiology, Mafraq Hospital, United Arab \\ Emirates \\ ${ }^{4}$ Division of Neurology, Mafraq Hospital, United Arab Emirates \\ Correspondence: Shobhit Sinha, Division of Neurology, \\ Mafraq hospital, Abudhabi, United Arab Emirates, \\ Emailssinha@mafraqhospital.ae \\ Received: November 22, 2014 | Published: December 18, \\ 2015
}

\section{Introduction}

Refractory status epilepticus (RSE) is defined as a persisting seizure activity (status epilepticus) that is unresponsive to initial standard first and second line anti-seizure therapy. ${ }^{1}$ It is a neurological emergency that requires early recognition and treatment.Most patients with RSE carry a high mortality and morbidity, and require treatment with multiple anticonvulsants including anesthetic agents. ${ }^{2}$

Management of RSE during pregnancy is even more challenging because of multiple reasons:

1. Two lives (mother $\&$ fetus) are at stake

2. Limited safe therapeutic options available during pregnancy

3. Changed pharmacokinetics of anti-seizure drugs during pregnancy

4. Limited safety and efficacy data available on prolonged use of anesthetic agents for treatment of RSE

We present a pregnant woman at 20 weeks of gestation who presented with RSE that necessitated the prolonged use of continuous intravenous infusion of the anesthetic sedative Propofol.

\section{Method}

We describe the clinical presentation, electro-imaging findings, management and clinical course of the 31 -year-old pregnant patient who presented to our hospital with RSE.

\section{Case presentation}

The patient, with prior intractable epilepsy since 22years, presented at 20 weeks of gestation. Her seizures were described as seeing left sided flashing light followed by impaired consciousness with head turning to the left side. This may be followed by secondary generalized tonic-clonic activity. She was on multiple anti-epileptic drugs (AEDs), and had Vagal Nerve Stimulator (VNS) implanted 5 years ago. In spite of that, her baseline seizure frequency was 2-3 times/month.
She was brought to the hospital with 2 days history of frequent focal seizures with intermittent generalizations. In between the seizures, she would be conscious and oriented. The seizures were believed to be precipitated by missing one dose of her AEDs and sleep deprivation for a few days. She denied any associated fever, chills, headache, or vomiting suggestive of ongoing infectious or inflammatory process.

Her medications were Lamotrigine (150 mg BID), Topiramate (200 mg BID), Levetiracetam (1500 mg BID), and Folic acid. The VNS Output Current was $2.75 \mathrm{~mA}$ (other settings- Frequency $30 \mathrm{~Hz}$; Pulsewidth- 500microseconds; On-time 30 Sec; Off-time 5 minutes).

On presentation to the Emergency Room, the vital signs were stable (weight- $59.6 \mathrm{~kg}$, blood pressure $121 / 60 \mathrm{mmHg}$, pulse rate $78 \mathrm{bpm}$, respiratory rate 18 per minute, oxygen saturation of $100 \%$ on room air and a temperature of 36.6 degree Celsius). The patient was having 10-15 clinical seizures/hour. These were characterized by behavior arrest; unforced head-turn to the right side, followed by the forced a head-turn to the left side and left side upper extremity jerking. In between seizures, a patient would be awake and responsive. She was oriented to place and person. Her cranial nerves exam was normal, and she was moving all 4 extremities well. Reflexes were normal apart from equivocal left Planter reflex. Pain and light touch sensation were appreciated bilaterally.

Labs including blood counts, liver \&renal functions, blood glucose\&electrolytes were normal. On presentation, serum drug level for Levetiracetamwas $8.10 \mathrm{mg} / \mathrm{L}$ (therapeutic range $5.80-17.80$ ), and Lamotrigine was $1.4 \mathrm{mg} / \mathrm{L}$ (3-14.0), a Limited Brain MRI study done on the 3rd day of admission showed mild right frontal cortical hyper-intensity on Diffusion Weighted Images (DWI), suggestive of probable seizure related changes.

The patient was given intravenous (IV) Lorazepam and Phenytoin (PHT) loading $(20 \mathrm{mg} / \mathrm{kg})$. PHT post-load level was $61.4 \mathrm{micromol} / \mathrm{L}$ (therapeutic range40-80). The VNS output current was increased to $3 \mathrm{~mA}$.Despite that, a seizure continued to progress in duration and frequency and eventually the patient was not regaining her consciousness between the attacks. Bedside EEG showed almost continuous right hemispheric seizure discharges that would frequently spread to involve the left side (Figure 1). The patient was intubated 
and IV Propofol was started with a bolus dose of $50 \mathrm{mg}$, followed by maintenance infusion of $200 \mathrm{mg} /$ hour that stopped the seizure activity within 30 minutes. The infusion was maintained at the same dose for next 48 hours. The patient during this time was under continuous bedside EEG monitoring. After 48 hours, the propofol was slowly tapered (by 50mg every hour) to stop. There was no electrographic or clinical seizure recurrence, and the patient was extubated. Electrographically, the EEG showed a generalized anesthetic pattern with intermittent, independently bi-temporal epileptogenic abnormalities (Figure 2).

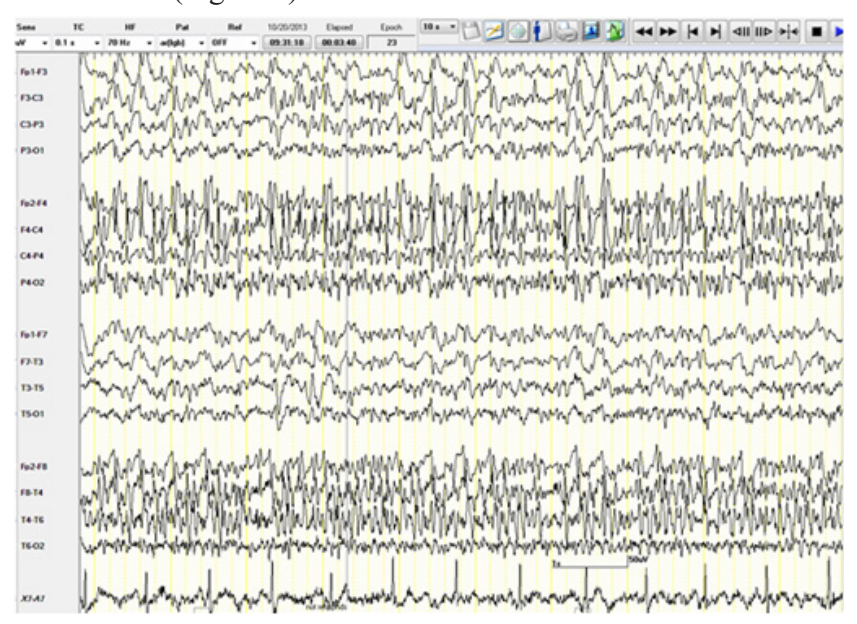

Figure I Bedside EEG showing continuous right hemispheric seizure discharges that would frequently spread to involve the left side.

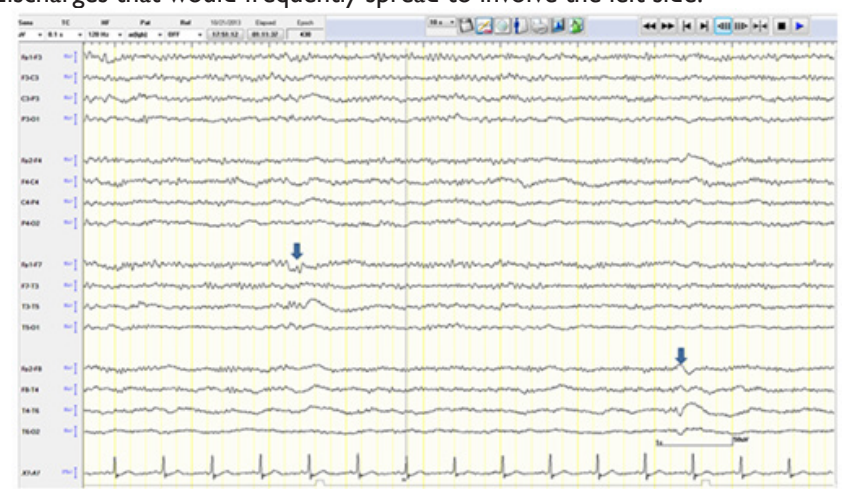

Figure 2 EEG showing a generalized anesthetic pattern with intermittent independent bi-temporal (arrows) epileptogenic abnormalities.

Fetal ultrasound prior, during and following Propofol infusion were unchanged and showed 22 weeks old viable fetus with normal heart-rate and movements. The patient remained seizure free for the next five days, and was discharged home on home AEDs and PHT $(300 \mathrm{mg} /$ day). Post-discharge, she continued to have intermittent mild focal seizures in spite of out-patient AED adjustments. Her antenatal follow ups were unremarkable, and she gave birth to a full term healthy baby.

\section{Discussion}

Status epilepticus (SE) in pregnancy is rare. According to the European Registry of Antiepileptic Drugs in Pregnancy (EURAP), SE occurred in $1.8 \%$ of the pregnancies. ${ }^{3}$ There is, however, lack of reliable statistics about the incidence of RSE in pregnancy. There have been only a few case reports of RSE during pregnancy and most of them were provoked by reversible metabolic or structural pathologies. ${ }^{4}$

The reasons why some women with epilepsy develop uncontrolled seizures during their pregnancy is still not well understood. Many studies conducted to evaluate the effects of pregnancy on epileptic women found that the rate of seizures in epileptic women increased during pregnancy ranged between $17-45 \% .{ }^{3,5}$ Several mechanisms may exacerbate epilepsy during pregnancy, including non-compliance, AEDs pharmacokinetics, hormonal changes, psychological and behavioral changes like sleep deprivation. ${ }^{6}$ The results of these studies showed profound inter-individual variability, and most studies were not case-controlled.

Serum concentration of most of the AEDs, some more than the other, decrease during pregnancy. This is probably related to the physiological changes in pregnancy that affects the intestinal absorption, volume of distribution, protein binding, drug metabolism, and renal clearance of these medications. The plasma concentrations of Lamotrigine and Leviteracetam may drop by $>50 \%,{ }^{8}$ and that could be a reason why our patient progressed to SE by missing a single dose.

European Federation of Neurological Societies (EFNS) guidelines on management of SE advocate proceeding immediately to the infusion of anesthetic doses of Midazolam, Propofol or barbiturates in RSE9.Lacking the evidence to support the use of specific anesthetic over the other, no recommendation is made regarding the choice of the drug. Depending on in-house protocol, drug is titrated to seizure cessation, burst suppression or complete suppression on the EEG. Following this, the infusion should be maintained for at least 24 hours. ${ }^{9,10}$ There is currently no data or guidelines for management of SE or RSE during pregnancy. This may be due to paucity of such cases.

Propofol has a short half-life, which allows rapid titration and withdrawal. It enhances the seizure inhibitory pathway through agonistic action on the GABA receptors. In addition, it has NMDA antagonistic action that inhibits the seizures excitatory pathway, and modulates $\mathrm{Na}$ and $\mathrm{Ca}$ channels. All of these make Propofol an excellent choice to treat RSE, but it carries the risk of Propofol infusion syndrome (PRIS), which consist of cardiocirculatory collapse with hypertrigleridemia, rhabdomyolysis, lactic acidosis and renal failure. However, PRIS is a potential problem when propofol is used in high infusion rate $(>4 \mathrm{mg} / \mathrm{kg} / \mathrm{hr})$ or long duration ( $>48$ hours). ${ }^{11}$ In our patient, we titrated the Propofol infusion to electrographic seizure cessation (rather than to burst- suppression or complete suppression), and started to taper the drug after 48 hours, thus minimizing the chances of PRIS.

Prolonged Propofol infusion to treat RSE in second trimester pregnancy has never been reported yet, but it has been reported for other anesthetic purposes with varied outcomes. ${ }^{12}$ It has been used in two neurosurgical pregnant patients, for a duration of less than 24 hours, and in both cases Propofol had to be substituted with another anesthetic agent as the patients developed mild acidosis, but no neonatal adverse events has been reported in both cases. ${ }^{13}$ Another case reported the use of Propofol as a sedative in a 2 nd trimester pregnant lady with lymphoma obstructing her airway, the duration of infusion was 51 days with 5 days of interruption, she also received chemotherapy during her hospitalization, the mother's condition improved but the fetus was found to be non-viable and the pregnancy was terminated. ${ }^{14}$

There has been 1 case report of successful treatment of Eclampsia induced RSE with Propofol. ${ }^{15}$ The patient, 23 years old with 38 weeks of gestation, presented with seizures that continued post-delivery and were refractory to benzodiazepines, $\mathrm{MgSO} 4$, barbiturates and phenytoin, but responded rapidly to Propofol. The patient received an Propofol infusion at a dose of $4 \mathrm{mg} / \mathrm{kg} / \mathrm{hr}$ after an initial bolus of $50 \mathrm{mg}$, which rapidly controlled her seizures. The infusion was tapered over 
the next 24 hours to $1.5 \mathrm{mg} / \mathrm{kg} / \mathrm{hr}$ and gradually stopped by 48 hours. Propofol is assigned to pregnancy category " $\mathrm{B}$ " by the FDA, as animal studies didn't show fetal harm. Studies in human pregnancy are lacking. Although it has been reported to have safe outcomes as an anesthetic agent during cesarean sections in some cases, ${ }^{12}$ but still the manufacture doesn't recommend it for obstetrical procedures. Other anesthetic agents used for treating RSE are designated category " $D$ ".

In patients with RSE, early recognition and treatment is the key for a better outcome. In a special scenario such as Pregnancy, Propofol may serve as an effective and relatively safer treatment option as it did for our patient. Further studies and experience in managing RSE in such special population would help outline the standard of care.

\section{Acknowledgments}

None.

\section{Conflicts of interest}

None.

\section{References}

1. Bleck TP. Refractory status epilepticus. Curr Opin Crit Care. 2005;11(2):117-120.

2. Novy J, Logroscino G, Rossetti AO. Refractory status epilepticus: a prospective observational study. Epilepsia. 2010;51(2):251-256.

3. Page B Pennell. EURAP outcomes for seizure control during pregnancy: useful and encouraging data. Epilepsy Curr. 2006;6(6):186-188.

4. Schulze-Bonhage A, Kurthen M, Walger P, et al. Pharmacorefractory status epilepticus due to low vitamin B6 levels during pregnancy. Epilepsia. 2004;45(1):81-84.

5. Knight AH, Rhind EG. Epilepsy and pregnancy: a study of 153 pregnancies in 59 patients. Epilepsia. 1975;16(1):99-110.
6. Schmidt D, Canger R, Avanzini G, et al. Change of seizure frequency in pregnant epileptic women. $J$ Neurol Neurosurg Psychiatry. 1983;46(8):751-755.

7. Harden CL, Hopp J, Ting TY, et al. Practice parameter update: management issues for women with epilepsy-focus on pregnancy (an evidence-based review): obstetrical complications and change in seizure frequency: report of the Quality Standards Subcommittee and Therapeutics and Technology Assessment Subcommittee of the American Academy of Neurology and American Epilepsy Society. Neurology. 2009;73(2):126-32.

8. Brodtkorb E, Reimers A. Seizure control and pharmacokinetics of antiepileptic drugs in pregnant women with epilepsy. Seizure. 2008;17(2):160-165.

9. Meierkord H, Boon P, Engelsen B, et al. European Federation of Neurological Societies. EFNS guideline on the management of status epilepticus in adults. Eur J Neurol. 2009;17(3):348-355.

10. Claassen J, Hirsch LJ, Emerson RG, et al. Treatment of refractory status epilepticus with pentobarbital, propofol, or midazolam: a systematic review. Epilepsia. 2002;43(2):146-153.

11. Iyer VN, Hoel R, Rabinstein AA. Propofol infusion syndrome in patients with refractory status epilepticus: an 11-year clinical experience. Crit Care Med. 2009;37(12):3024-3030.

12. Kariya N, Toyoyama H, Furuichi K, et al. Induction of general anesthesia using propofol for cesarean section of a woman with cerebral palsy. $J$ Clin Anesth. 1999;11(8):672-674.

13. Hilton G, Andrzejowski JC. Prolonged propofol infusions in pregnant neurosurgical patients. J Neurosurg Anesthesiol. 2007;19(1):67-68.

14. Tajchman SK, Bruno JJ. Prolonged propofol use in a critically ill pregnant patient. Ann Pharmacother. 2010;44(12):2018-2022.

15. Dam Ak, Mishra JC, Shome PK. Treatment of refractory seizures in eclampsia with propofol- a case report. Indian $J$ Anaesth. 2004;48(4):314-315. 